Historic, archived document

Do not assume content reflects current scientific knowledge, policies, or practices. 
62,93

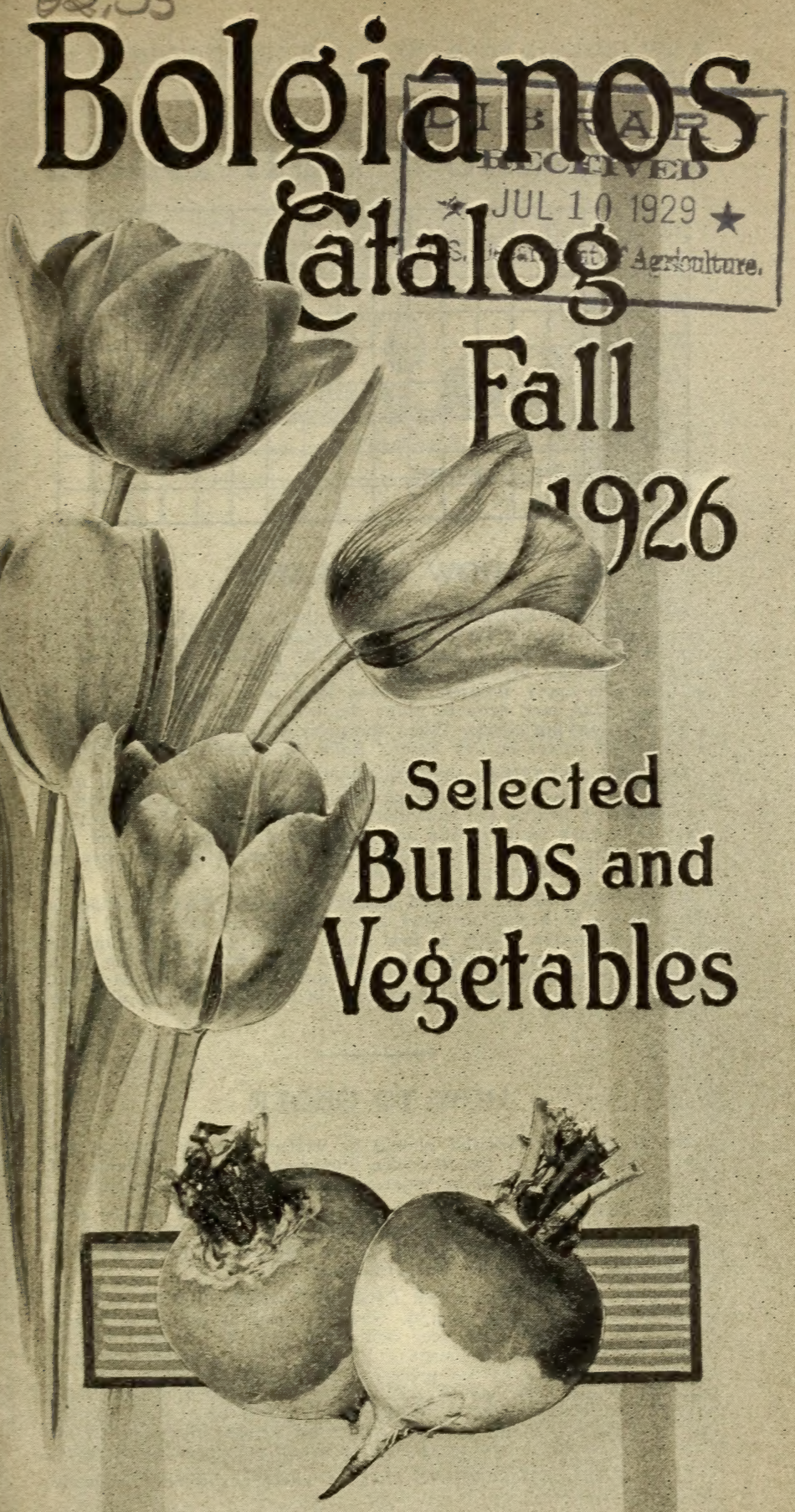

F.W. Bolgiano \& Co. "Capitol City Seeds" Washington, D.C. 


\section{Bolgiano's Fall Bulbs}

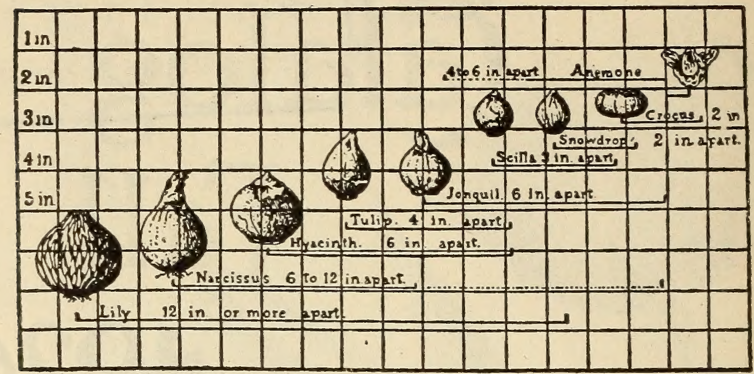

\section{HOW TO PLANT}

OUTDOOR CULTURE-Plant the bulbs as soon after September as possible to enable the roots to make good growth before the cold weather sets in. They can, however, be planted from September until the ground becomes frozen. They require a moderately rich soil, or else apply Bolgiano's Sheep Manure or Bone Meal. How far apart and how deep to plant the different bulbs is shown in the accompanying chart. When the ground freezes cover with 3 or 4 inches of leaves or dry litter. Rake off after frost.

INDOOR CULTURE-Plant hyacinths, tulips and narcissus in a sandy soil. Give a good soaking after planting and water once or twice a week during the time the bulb makes root. After planting keep in a dark place in the house and keep a paper beg over the pot until the sprout is about three inches long. They may then be brought to the light and bag removed.

GROWING PAPER WHITE NARCISSUS IN WATER-Place a dozen bulbs in a shallow bowl, cover with small stones and keep filled with water. Place a few small pieces of charcoal in the bottom of the bowl to keep the water from fouling. Keep in a moderately temperate room. They require no care, no attention. They keep on growing and produce a beautiful flowering plant in about six weeks.

\section{HOW TO ORDER}

NAME AND ADDRESS should be written plainly both on the Order Blank and on the envelope enclosing the order. Names of seeds and bulbs should be written out in full in plain writing.

OUR TERMS are always cash with order. MONEY can be sent at our risk by Post Office or Express Money Order, New York or Chicago Draft, or by registered letter. We cannot hold ourselves responsible for cash sent loose in letters.

POSTAGE AND EXPRESS CHARGES.-ALL BULBS will be sent by Mail charges prepaid, when ordered at single or dozen rates. At 100 rates and over add 5 per cent to the cost of the bulbs to cover carrying charges. Packets, Ounces, Quarter-Pounds or Pounds, ordered at list prices, will be sent FREE by mail or express. This DOES NOT APPLY to Beans, Corn, Peas, Field and Grass Seeds and Seed Potatoes, which will be sent at regular Parcel Post or Express Rates. Orders for greater than one pound lots will also be delivered at regular parcel post or express rates.

GIVE FULL DIRECTIONS always, whether shipment is made by freight, express or Parcel Post.

EXPRESS OR FREIGHT shipments should have the name of the station plainly given where different from the post-office.

WE GUARANTEE our seeds, plants, etc., to reach our customer in good condition. We give no warranty, expressed or implied, as to description, purity or productiveness, or any other matter of any seeds, plants or bulbs we send out, and will not be in any way responsible for the crop. If purchaser does not accept the goods on these terms they are to be returned at once, and the noney that has been paid for same will be refunded. 


\section{SINGLE HYACINTHS}

Ready for Delivery late in September

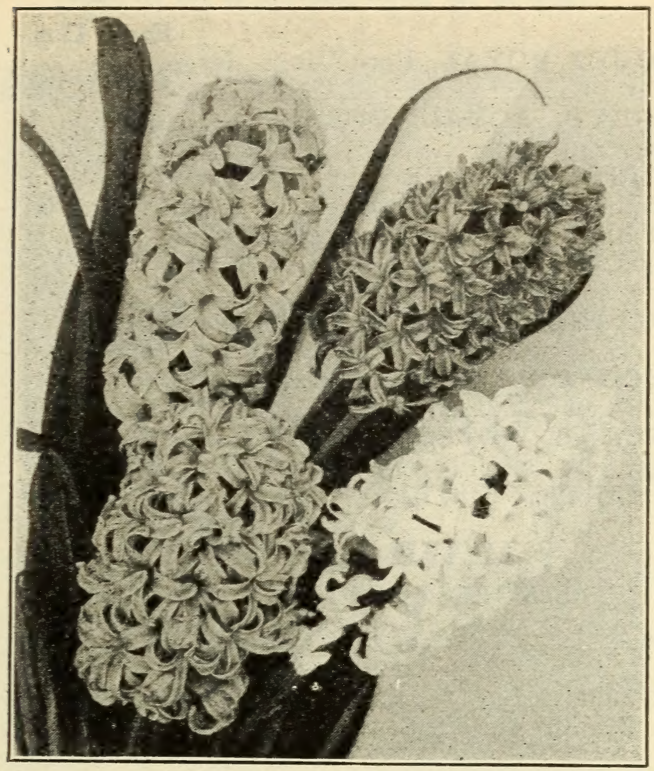

FIRST SIZE-EXTRA SELECTED

6 Sold at the Dozen Rate, 25 at the 100 Rate

Each. Doz. 100.

BLANCHEUR A MERVEILLE, Pure

White, good spikes with large bells........\$0.20 \$2.25 \$17.00

GERTRUDE, Light Red, compact spikes $.20 \quad 2.25 \quad 17.00$

GIGANTEA, Blush Pink, large, broad spike

$\begin{array}{lll}.20 & 2.25 & 17.00\end{array}$

GRAND MAITRE, Fine Deep Porcelain

Blue, broad, well formed spike.

JOHAN, Light Blue, strong bells..

$\begin{array}{lll}.20 & 2.25 & 17.00\end{array}$

KING OF THE BLUES, Deep Glossy

Blue, medium size bells.

L'IN NOCENCE, Pure White, large, well

shaped spikes

MAD. VANDERHOOP, Almost Pure

White, and if forced, pure white.............

LA VICTOIRE, Bright Scarlet, compact truss of medium size bulbs.

YELLOW HAMMER, Pure Yellow.

$$
\text { SECOND SIZE }
$$

$.20 \quad 2.25 \quad 17.00$

$\begin{array}{lll}.20 & 2.25 & 17.00\end{array}$

$\begin{array}{lll}.20 & 2.25 & 17.00\end{array}$

$\begin{array}{lll}.20 & 2.25 & 17.00\end{array}$

$\begin{array}{lll}.20 & 2.25 & 17.00\end{array}$

$\begin{array}{lll}.20 & 2.25 & 17.00\end{array}$

BLANCHEUR A MERVEILLE

GERTRUDE $\$ 0.18 \$ 1.85 \$ 14.50$

GIGANTEA

$.18 \quad 1.85 \quad 14.50$

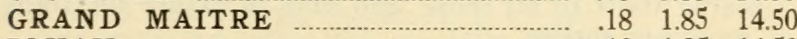

JOHAN

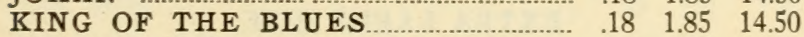

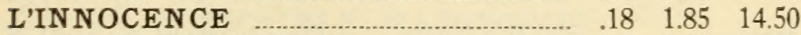

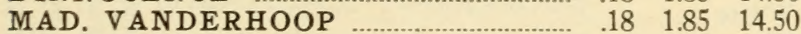

LA VICTOIRE

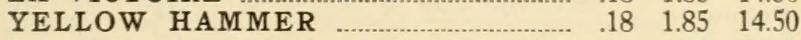

NOTE-We pay postage on all Bulbs at the each and dozen rate. At the 100 rate add 5 per cent to the cost of the Bulbs for carrying charges. 


\section{DOUBLE HYACINTHS-First Size}

Ready for Delivery late in September

6 Sold at the Dozen Rate, 25 at the 100 Rate

BOUQUET ROYAL, Pure White, large

Each. Doz. 100.

spike

$\$ 0.18 \$ 2.00 \$ 15.00$

BOUQUET ROYAL ROSE, Salmon

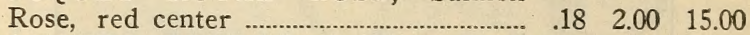

BOUQUET TENDRE, Brilliant Dark

Carmine Red, good truss........................... $18 \quad 2.00 \quad 15.00$

BLOKSBERG, Light Blue, large spike $\begin{array}{llll}.18 & 2.00 & 15.00\end{array}$

GENERAL KOHLER, Bright Blue,

large spike …….............................................

LA GRANDESSE, Pure White, waxy

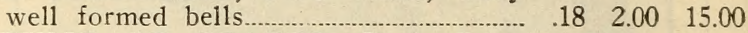

PRINCE OF ORANGE, Dark Rose,

large truss .........

large bells, slightly rosy center.................. $.18 \quad 2.00 \quad 15.00$

$\begin{array}{lllll}\text { VAN SPEYK, Light Blue, very large bells } & .18 & 2.00 & 15.00\end{array}$

\section{MIXED HYACINTHS}

\section{SINGLE BEDDING VARIETIES}
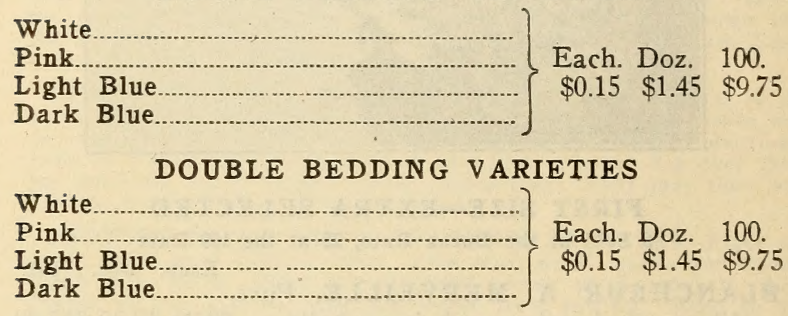

\section{CROCUS}

Ready for Delivery late in September

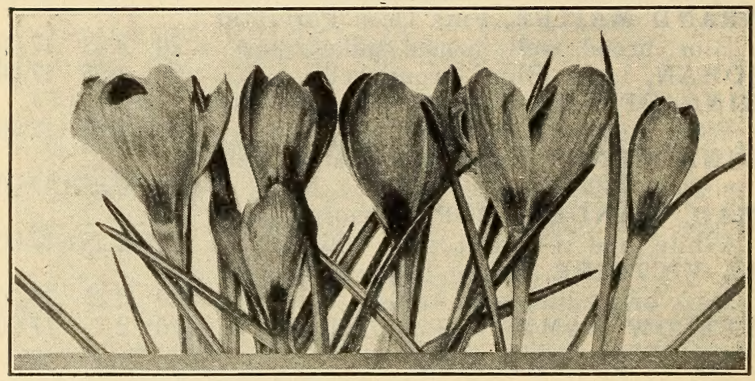

The earliest of flowers to show its head among the grass almost before the snow is gone, this little gem has endeared itself to all those who look to nature for signs of returning spring.

EXTRA LARGE SIZE

White

Each. Doz. 100.

Blue $\$ 0.04 \$ 0.30 \$ 1.75$

Yellow

$.04 \quad .30 \quad 1.75$

Striped

$.04 \quad .30 \quad 2.00$

NOTE-We pay postage on all Bulbs at the each and dozen rate. At the 100 rate add 5 per cent to the cost of the Bulbs for carrying charges. 


\section{NARCISSUS AND JONQUILS}

Ready for Delivery late in September

6 Sold at the Dozen Rate, 25 at the 100 Rate

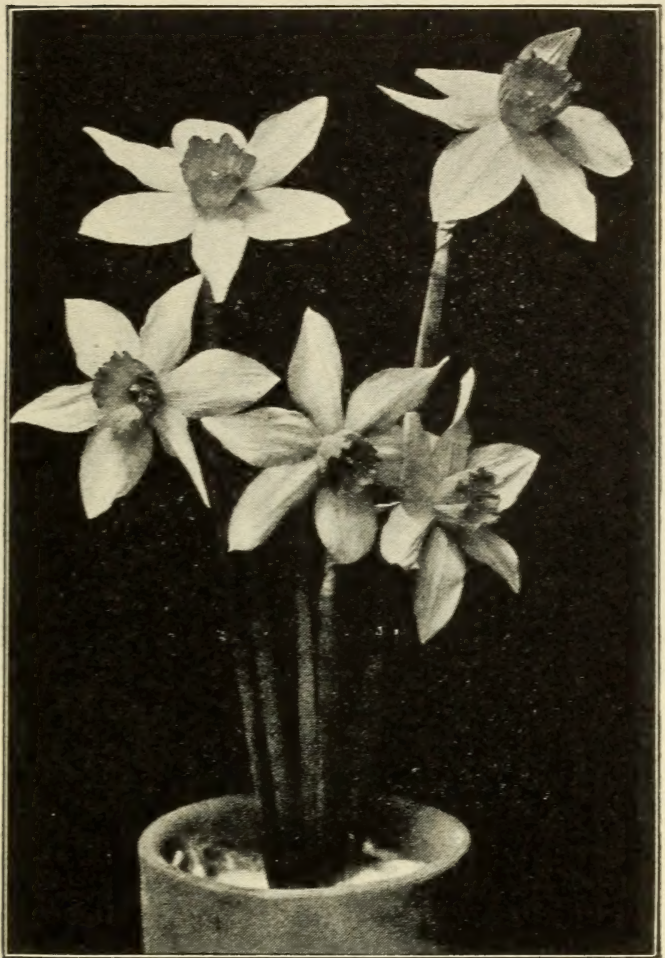

\section{SINGLE NARCISSUS}

\section{LARGE TRUMPET VARIETIES}

Each. Doz. 100.

BICOLOR VICTORIA, Petals creamy white, trumpet rich yellow, unsurpassed for forcing, extra double nose bulbs..................... $\$ 0.15 \quad \$ 1.50 \quad \$ 11.50$ EMPEROR, A magnificent gigantic flower having an immense trumpet of clear golden yellow......... $.15 \quad 1.50 \quad 11.50$ SIR WATKINS, one of the best Yellow with white Derianth, extra double nose bulbs............ $15 \quad 1.50 \quad 11.50$ GOLDEN SPUR, Rich Golden Yellow best single trumpet market daffodil, extra double nose bulb. $.15 \quad 1.50 \quad 11.50$ PRINCEPS GIANT, Petals pale sulphur yellow, trumpet yellow, extra double nose bulbs........... $10 \quad 1.00 \quad 7.50$

MEDIUM AND SMALL TRUMPET VARIETIES POETICUS RECURVUS, Pheasant's Eye, pure white petals, cup yellow with red, extra double nose bulbs

\section{DOUBLE NARCISSUS}

VON SION, Yellow trumpet and petals, large flowers, splendid for forcing or bedding, extra

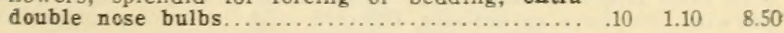
NOTE-We pay postage on all Bulbs at the each and dozen rate. At the 100 rate add 5 per cont to the cost of the Bulbs for carrying charges. 


\section{SINGLE EARLY TULIPS}

Ready for Delivery late in September 6 Sold at the Dozen Rate, 25 at the 100 Rate

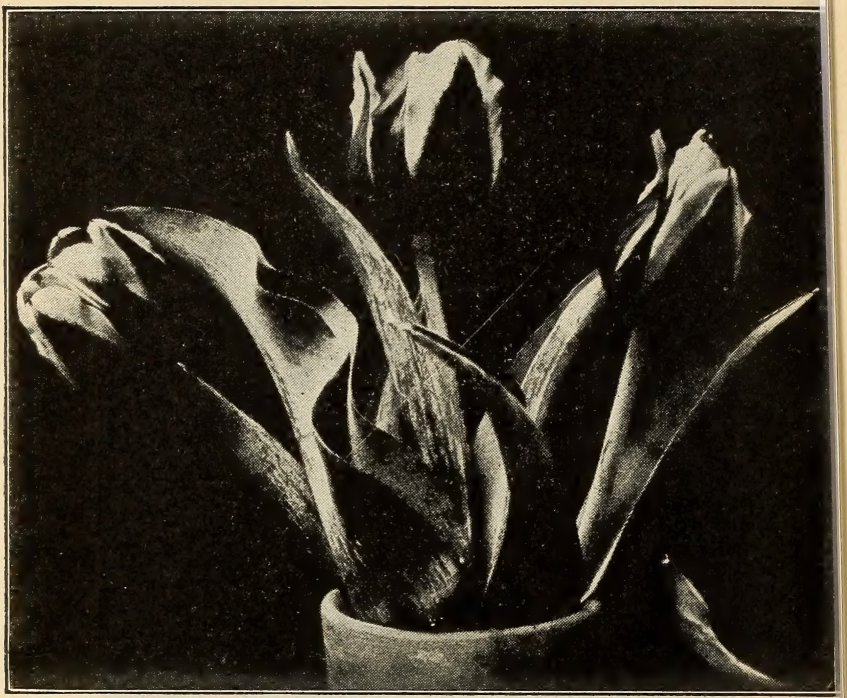

ARTUS, Dark Scarlet, large flower.

Each. Doz. 100. CHRYSOLORA, Large Pure Yellow $\$ 0.06 \$ 0.60 \$ 4.65$

COTTAGE MAID, Dark Rose shaded with White $\begin{array}{lll}.07 & .70 & 5.25\end{array}$

DUCHESS DE PARMA, Brownish Red with large light orange border.

KEIZERKROON, Bright Red with broad golden yellow border.

$\begin{array}{lll}.06 & .60 & 4.50\end{array}$

$\begin{array}{lll}.06 & .65 & 5.00\end{array}$

IA REINE (Queen Victoria), White, sometimes showing a rose tint.................. $\quad .06 \quad .60 \quad 4.65$

POTTEBAKKER SCARLET, Large scarlet flower ……........................................... $.06 \quad 60 \quad 4.65$

POTTEBAKKER WhITE, Pure White, fine, large form..

ROSE GRIS-DE-LIN, Delicate Rose shading to white at base

YELLOW PRINCE, Bright Yellow splendid forcer

\section{MIXED SINGLE TULIPS}

Red, Pink, Yellow, White.

\section{MIXED DOUBLE TULIPS}

Red, Pink, Yellow, White

\section{OLD DUTCH or BREEDER TULIPS}

This fine old May Flowering type is exceedingly popular. They are very similar to the Darwins in their tall strong growth but with even larger flowers. They bloom about the same time as the Darwins, but have an entirely different range of colors, comprising Bronze, Terra-cotta, Orange, Brown and other artistic tones.

Old Dutch, or Breeder Tulips Mixed...... $\$ 0.05 \quad \$ 0.55 \$ 4.50$ 


\section{DARWIN TULIPS}

Ready for Delivery late in September 6 Sold at the Dozen Rate, 25 at the 100 Rate

CLARA BUTT, Bright Salmon Rose, Each. Doz. 100. lovely color and shape.... $\$ 0.05 \$ 0.50 \$ 3.25$

FARNCOMBE SANDERS, Brilliant Rose Scarlet
GRETCHEN (Margaret), Soft Flesh Rose, beautiful shape......... KRELAG, Bright Rose, light

MAD. KRELAGE, Bright Rose, light
border, fine, large flower................................ PAINTED LADY, Creamy White, faintly tinged heliotrope

PRIDE OF HAARLEM, Brilliant Carmine Rose, very large..

REV. H. EWBANK, Bright Lavender, splendid for early forcing...................... ZULU, Rich Velvety Purple Black, very fine distinct shape............... perior Mixture

$$
\begin{array}{lll}
.06 & .70 & 5.00
\end{array}
$$$$
.06 \quad .60 \quad 4.00
$$$$
.06 \quad .60 \quad 4.50
$$

$.06 \quad .60 \quad 4.50$

$\begin{array}{lll}.05 & .50 \quad 4.00\end{array}$

$.06 \quad .60 \quad 4.50$

$.06 \quad .60 \quad 4.65$

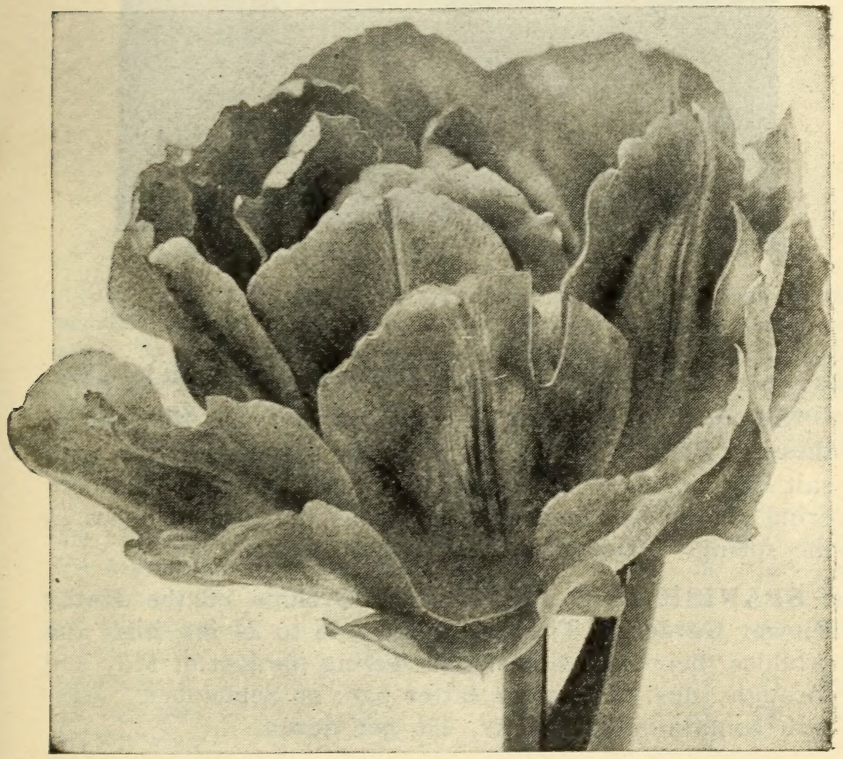

\section{DOUBLE EARLY TULIPS}

Ready for Delivery late in September

LA CANDEUR, White, green tipped,

Each. Doz. 100. large, full double flower..........................\$0.06\$0.60\$4.65 MURILLO, White, shading to delicate pink, beautiful shape. IMPERATOR RUBRORUM, Pure

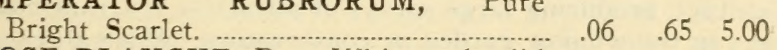
ROSE BLANCHE, Pure White, splendid for bedding TITIAN, Red with golden border, a double Keizerkroon, better than Tournesol NOTE-We pay postage on all Bulbs at the each and dozen rate. At the 100 rate add 5 per cent to the cost of the Bulbs for carrying charges. 


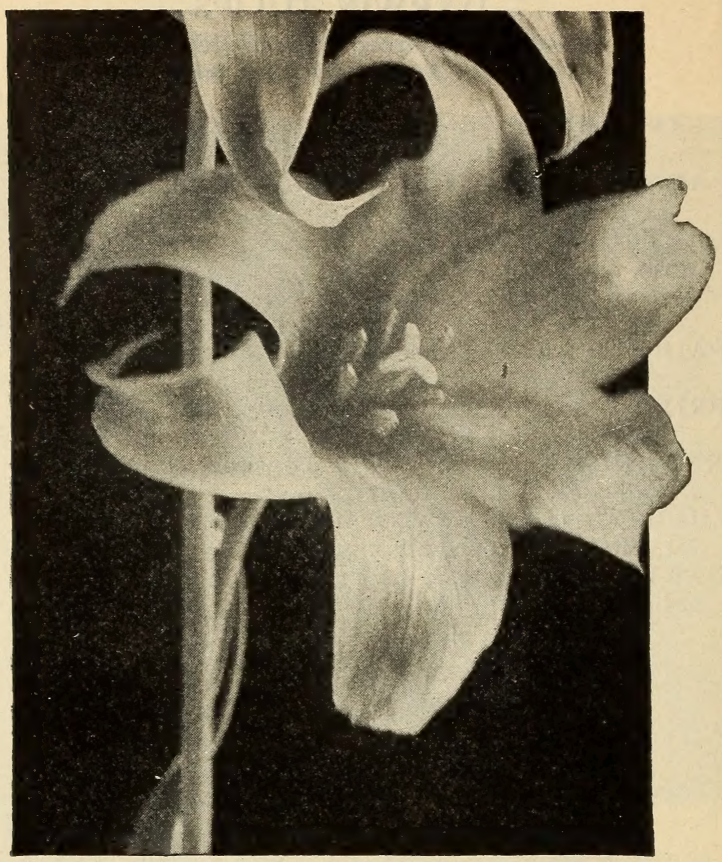

Easter I.ily.

\section{EASTER LILY (Lilium Formosum)}

"Solomon in all his glory was not arrayed like one of these." Plant 5 inches deep in the fall and cover with salt hay, leaves or debris about 4 inches thick. This covering should be taken off as soon as the ground opens in the spring. Prices, 25c each; $\$ 2.50$ dozen.

SPANISH IRIS. Known as "Orchids of the Hardy Flower Garden." They grow from 18 to 24 ins. high and produce their exquisite blooms during the last of May and through June. Plant the latter part of September. Mixture containing all colors, $75 \mathrm{c}$ per dozen.

\section{INDOOR FLOWERS FOR WINTER BLOOMS}

CALLA LILY (Lily of the Nile). Plant in a 6-inch pot in September, give plenty of water, light and heat, and your reward will be large blooms of purest white. Very easy to grow. $25 \mathrm{c}$ each; $\$ 2.75$ per doz.

FREESIAS, RAINBOW MIXTURE. Popular for pot culture, producing large sprays of attractive fragrant flowers in the window garden or conservatory. Plant three or four bulbs in a 6-inch pot. 70c doz.; $\$ 5.00$ per 100 .

OXALIS. Large bright flowers over dwarf sturdy growth. Blooms profusely from November to June. Mixture of all colors, 5c each; 50c per doz. 


\title{
FLOWER SEEDS FOR FALL PLANTING
}

\author{
POSTAGE PAID
}

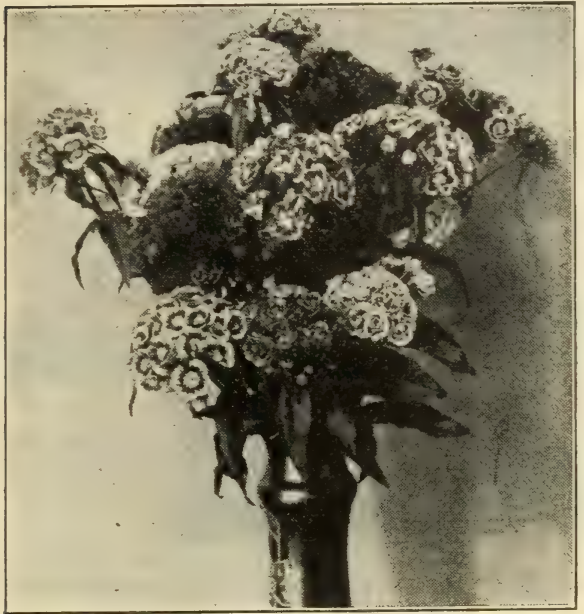

Sweet William

\section{THE HARDY PERENNIAL GARDEN}

Year after year the hardy perennial garden is increasing in popularity. From early in April they continue to bloom with constant changing variety throughout the entire Summer and Fall. Once planted they appear themselves each year and do away with the necessity of annual seeding. Sown in late Summer or early Fall bloom next year. No garden is complete without a liberal space devoted to Hardy Perennials.

Pkt. $1 / 4 \mathrm{Oz} . \mathrm{Oz}$.

\begin{tabular}{|c|c|c|c|}
\hline & & & \\
\hline 9 & Aquilegia (Columbine Mixed) & .35 & 1.00 \\
\hline & ampanula & .25 & 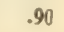 \\
\hline & & 0 & \\
\hline & …… & & \\
\hline & $\ldots \ldots \ldots \ldots \ldots \ldots$ & .25 & .85 \\
\hline & .1 & .20 & .55 \\
\hline & & & \\
\hline & pur........... .1 & .75 & \\
\hline & s, Pheasant's Eye Pink. & .35 & 1.00 \\
\hline & ... . .10 & .75 & \\
\hline & . & 5 & 1.00 \\
\hline & 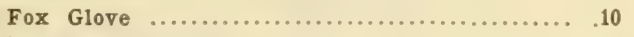 & .25 & .7 \\
\hline & .1 & .25 & .7 \\
\hline & .1 & .20 & .75 \\
\hline & cck & .30 & 1.00 \\
\hline & $\cdots . .1$ & & .7 \\
\hline & … .10 & .85 & 3.0 \\
\hline & Sy Giant Ado & .90 & 3.2 \\
\hline & red Trimardeau Mixed....... .1 & .90 & 3.0 \\
\hline & Giant Golden Yellow Purple Stained.... .10 & .90 & 3.25 \\
\hline & 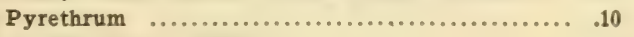 & .25 & .85 \\
\hline & 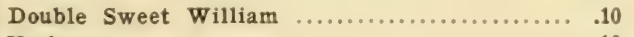 & .35 & 1.00 \\
\hline & ena $\ldots . . . . . . .$. & .40 & 1.2 \\
\hline & ower & .20 & \\
\hline
\end{tabular}




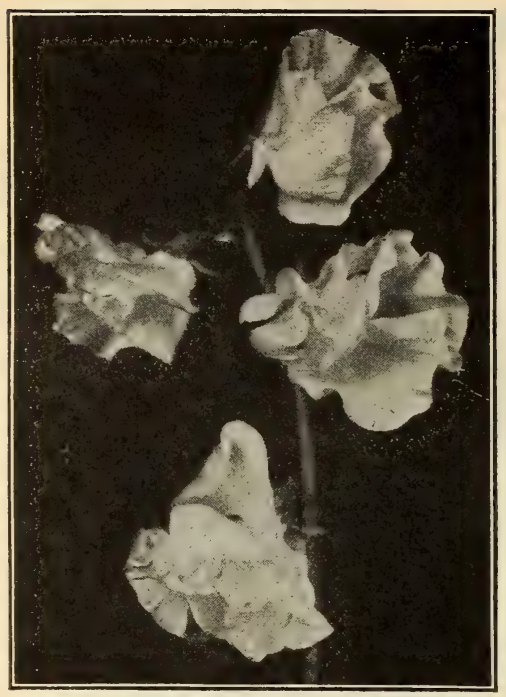

SWEET PEAS

Splendid results are often obtained from $\mathrm{g} r$ ow ing Sweet Peas in October or November. A perfectly drained soil so situated as not to be subject to too frequent or s e vere freezing should be selected, and the seed covered about three inches.

\section{SUPERB EARLY OR CHRISTMAS FLOWERING SPENCER SWEET PEAS}

These varieties are a welcome addition to our lists, for they flower several weeks earlier than the regular Spencers, and if planted with them you will have a much longer season of bloom.

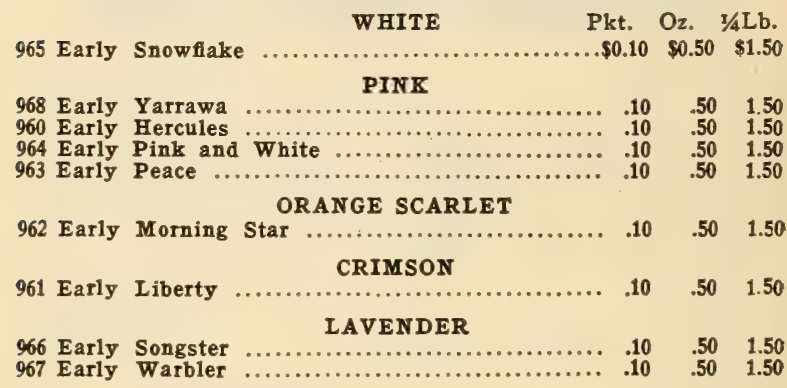

\section{SPENCER SWEET PEAS}

WHITE

Pkt. Oz. $1 / 4 \mathrm{Lb}$. Lb.

986 King White ...........................\$0.10 \$0.25 \$0.95 \$3.25

973 Constance Hinton ........................ $10 \quad .25 \quad .95 \quad 3.25$

CREAM OR YELLOW

972 Clara Curtis ....................... .10 $\quad .25 \quad .95 \quad 3.25$

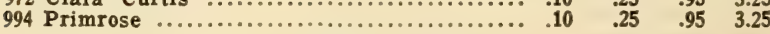

CREAM PINK

(Delicate Pink on Cream Ground)

987 Margaret Atlee ...........................10

991 Mrs. Routzahn ............................ 10

$\begin{array}{lll}.25 & .95 & 3.25 \\ .25 & .95 & 3.25\end{array}$

LIGHT PINK
977 Elfrida Pearson $\ldots \ldots \ldots \ldots \ldots \ldots \ldots \ldots \ldots$

DEEP PINK

974 Countess Spencer .......................10

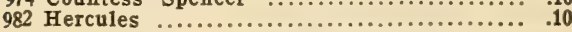

$.25 \quad .95 \quad 3.25$

SALMON PINK

976 Doris Usher

ROSE

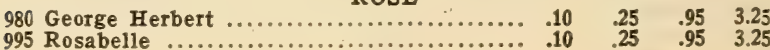




\section{SWEET PEAS}

Sweet Peas have been frequently referred to as the poor man's Orchid solely because of the abundance of blooms, the colors which may be likened to those found in the rarest Orchids.

\section{SPENCER SWEET PEAS}

ORANGE Pkt. Oz, $1 / 4 \mathrm{Lb}$. Lb.

978 Firey Cross ......................... \$0.10 $\$ 0.25 \quad \$ 0.95 \quad \$ 3.25$

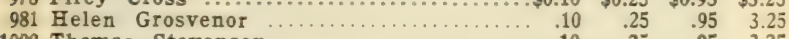

1000 Thomas Stevenson ...................... $10 \quad .25 \quad \begin{array}{llll}.95 & 3.25\end{array}$

SALMON

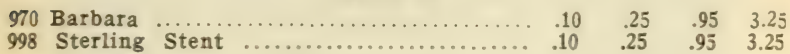

\section{LAVENDER}

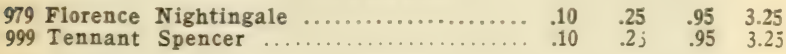

\section{BLUE}

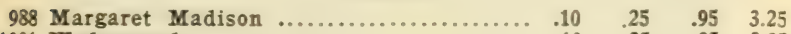

1001 Wedgewood ................................. $10 \quad .25 \quad .95 \quad 3.25$

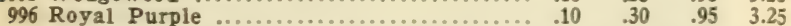

\section{MAROON}

993 Nubian $\ldots \ldots \ldots \ldots \ldots \ldots \ldots \ldots \ldots \ldots \ldots \ldots \ldots \ldots \ldots . .10 \quad .25 \quad .95 \quad 3.25$

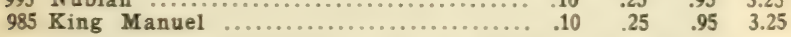

BI-COLOR (Pink and White)

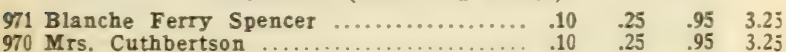

\section{PICOTTE EDGED}

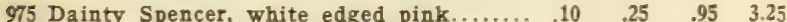

992 Mrs. Townsend, white edged blue............

989 Mrs. C. W. Breadmore, cream edged pink $\quad .10 \quad \begin{array}{lllll}.25 & .95 & 3.25\end{array}$

\section{STRIPED OR VARIEGATED}

997 Senator Spencer $\ldots \ldots \ldots \ldots \ldots \ldots \ldots \ldots \ldots \ldots \ldots .10 \quad .25 \quad .95 \quad 3.25$

1002 Superb Mixed Spencers $\ldots \ldots \ldots \ldots \ldots \ldots \ldots . . .10 \quad .20 \quad .60 \quad 2.00$

\section{GRANDIFLORA SWEET PEAS}

WHITE Pkt. Oz. $2 / 4 \mathrm{Lb} . \mathrm{Lb}$.

950 Dorothy Eckford $\begin{array}{lll}\$ 0.10 & \$ 0.15 \quad \$ 0.45 \quad \$ 1.50\end{array}$

YELLOW

952 Hon. Mrs. E. Kenyon ................ $10 \quad .15 \quad .45 \quad 1.50$

IIGHT PINK

956 Prima Donna .......................... $10 \quad .15 \quad .45 \quad 1.50$

DARK PINK

953 Janet Scott

ROSE

957 Prince of Wales ....................... $10 \quad .15 \quad .45 \quad 1.5$ J

CRIMSON OR SCARIET

$9 \Xi 4$ King Edward VII $\ldots \ldots \ldots \ldots \ldots \ldots \ldots \ldots \ldots \ldots .10 .15 \quad .45 \quad 1.50$

\section{LAVENDER}

955 Lady Grisel Hamilton

\section{MAROON}

948 Black Knight $\ldots \ldots \ldots \ldots \ldots \ldots \ldots \ldots \ldots \ldots \ldots \ldots . .10 \quad .15 \quad .45 \quad 1.50$

BI-COLOR (Pink and White)

949 Blanche Ferry .................... .10

\section{STRIPED OR VARIEGATED}

951 Helen Pierce .......................... .10 


\section{BOLGIANO'S "CAPITAL CITY" GARDEN SEEDS}

FOR FALL PLANTING

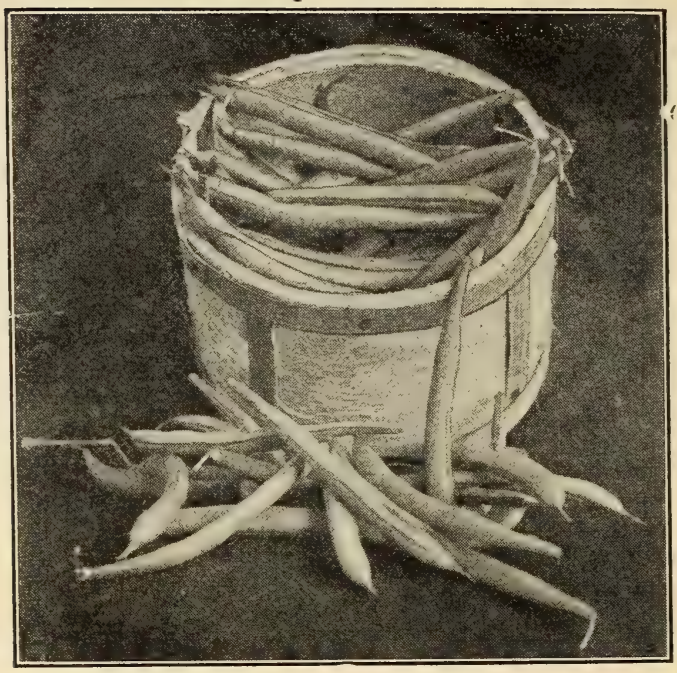

\section{BEANS}

REFUGEE (1,000 to 1). (45 days.) One of the most popular and attractive beans we have, especially adapted for Fall truck and market growing. Will stand light frosts without injury. The pods are about 7 inches long, nearly straight, round. They are brittle and of fine Havor. The plants are strong, vigorous growers and very productive. Pkt. $10 \mathrm{c}$; pt. $25 \mathrm{c}$; qt. $45 \mathrm{c}$; pk. $\$ 2.85$; bu. $\$ 10.50$;

$$
\text { Pt. Qt. Pk. Bu. }
$$

Stringless Refugee Greenpod............\$0.25 \$0.45 \$2.85 \$10.50

Black Valentine

$\begin{array}{llll}.25 & .45 & 2.75 & 10.00\end{array}$

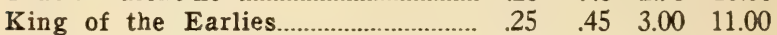

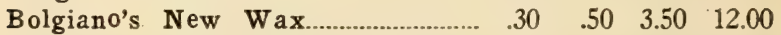

$\begin{array}{llllll}\text { Improved Rust-Proof Golden Wax } & .25 & .45 & 3.00 & 11.00\end{array}$

Improved Stringless Refugee Wax $\quad .30 \quad \begin{array}{llll}.50 & 3.50 & 12.00\end{array}$

$\begin{array}{lllll}\text { Wardwell's Kidney Wax........................ } & .25 & .45 & 3.00 & 11.00\end{array}$

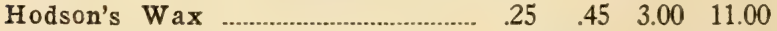

$\begin{array}{lllllll}\text { Currie's Rust-Proof Wax.................... } & .25 & .45 & 3.15 & 11.50\end{array}$

DAVIS WAX. The bush makes a vigorous, strong, upright growth, and is resistant to blight and rust. The pods are fleshy, of good length and excellent color. They are remarkable for their fine flavor, are very brittle and tender. A robust grower and a heavy yielder. Pkt. 10c; pt. $25 \mathrm{c}$; qt. $45 \mathrm{c}$; pk. $\$ 2.75$; bu. $\$ 10.00$.

KENTUCKY WONDER, or OLD HOMESTEAD POLE BEAN. Pt. 30c; qt. 50c; pk. $\$ 3.50$; bu. $\$ 12.00$.

BOLGIANO'S ENORMOUS BUSH LIMA. Pt. 50c; q.t. $\$ 1.00$; pk. $\$ 4.25$; bu. $\$ 16.00$.

F OR D H O K BUSH LIMA. Pt. 50c; qt. $\$ 1.00$; Fk. $\$ 4.25$; bu. $\$ 16.00$. 


\section{BEETS}

Superb

Crosby Egyptian

Pkt. Oz. T/4lb. Lb.

Detroit Dark Red

$\$ 0.10 \$ 0.15 \$ 0.35 \$ 1.25$

$\$ 0.10 \$ 0.15 \$ 0.30 \$ 0.85$

Deep Blood Beauty

$\begin{array}{llll}.10 & .15 & .30 & .85\end{array}$

$\begin{array}{lllll}\text { Eul Early Spring } & .10 & .15 & .35 & 1.25\end{array}$

$\begin{array}{lllll}\text { Eclipse } \ldots \ldots \ldots \ldots \ldots \ldots \ldots \ldots \ldots \ldots & .10 & .15 & .30 & .85\end{array}$

$\begin{array}{llllll}\text { Crimson Globe …………………… } & .10 & .15 & .20 & .65\end{array}$

Lentz

$\begin{array}{llll}10 & .15 & .20 & .65\end{array}$

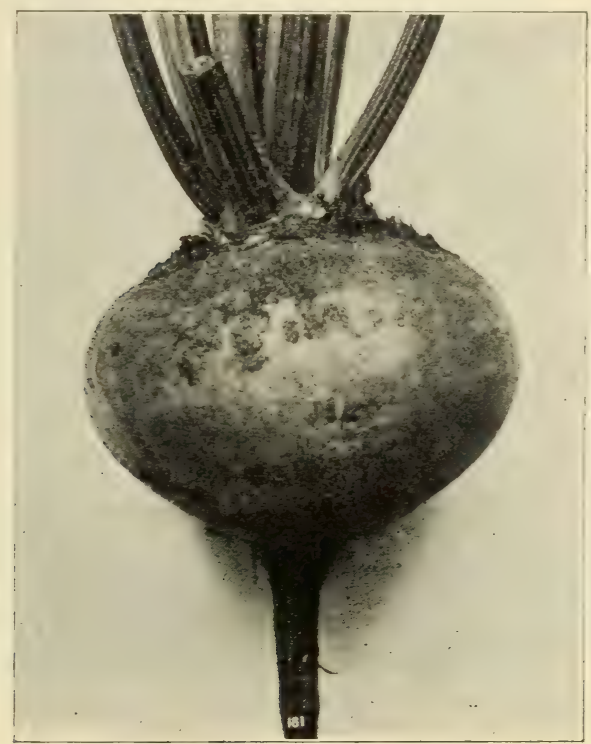

CAULIFLOWER

EARLY SNOWBALL. Pkt. $10 \mathrm{c}$ and $15 \mathrm{c} ; \mathrm{I} / 2 \mathrm{oz} . \$ 1.25$; 0z. $\$ 2.00 ; 1 / 4$ lb. $\$ 7.50$.

DWARF ERFURT. Pkt. $10 \mathrm{c}$ and $15 \mathrm{c} ; \mathrm{r} / 2$ oz. $\$ 1.40$; Oz. $\$ 2.25 ; \quad 1 / 4$ lb. $\$ 8.00$.

AUTUMN GIANT. Pkt. $10 \mathrm{c}$ and $15 \mathrm{c} ; \mathrm{T} / 2$ oz. 50c; Oz. $75 \mathrm{c} ; \mathrm{I} / 4$ lb. $\$ 2.70$; 1b. $\$ 10.00$.

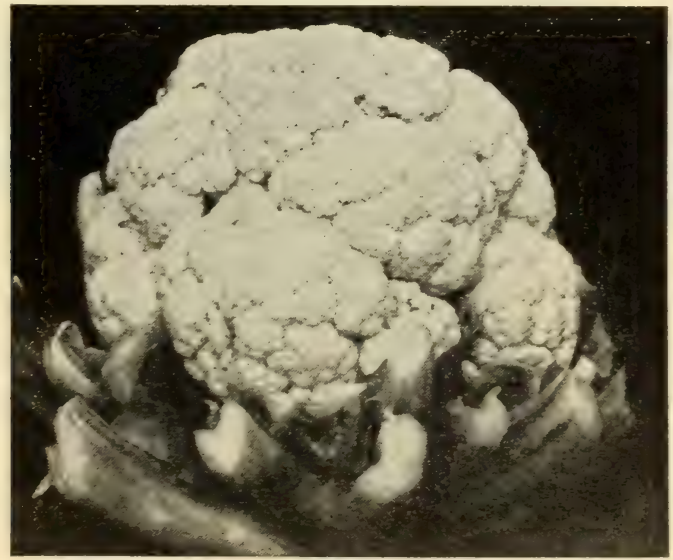

Early Snowball Cauliflower

(13) 
F. W. BOLGIANO \& CO. WASHINGTON, D. C.

\section{CELERY}

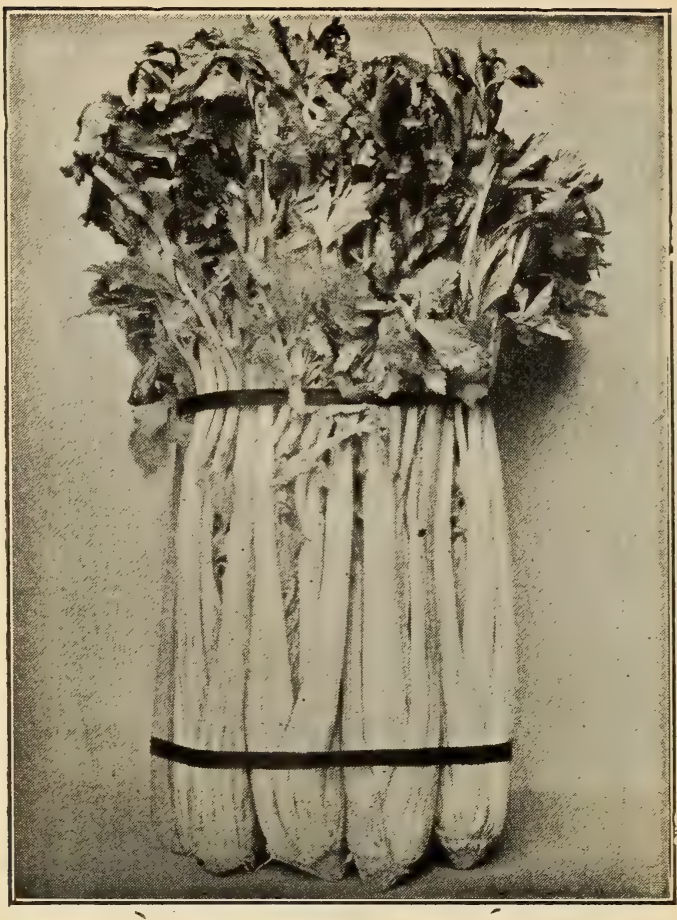

Bolgiano's Famous Stock Golden Self Blanching Celery.

Pkt. Oz. $1 / 4 \mathrm{lb}$. Lb.

Golden Self-Blanching $\$ 0.10 \$ 0.60 \$ 2.10 \$ 8.00$

Easy Blanching $\begin{array}{llll}.10 & .30 & 1.00 & 3.50\end{array}$

Sweet Nut

$\begin{array}{llll}.10 & .15 & .50 & 1.75\end{array}$

Giant Pascal

$\begin{array}{llll}.10 & .15 & .35 & 1.25\end{array}$

CELERIAC, GIANT SMOOTH PRAGUE

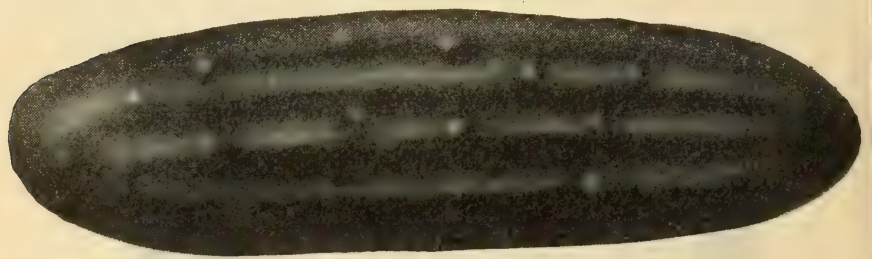

Bolgiano's Early Fortune Cuke.

\section{CUCUMBER}

Pkt. Oz. $1 / 4$ lb. Lb.

Bolgiano's Prosperity White Spine....\$0.10 $\$ 0.15 \$ 0.35 \$ 1.25$

Bolgiano's Early Fortune $\ldots$

$\begin{array}{lllll}\text { Klondyke …............................................................. } & .10 & .15 & .30 & .95\end{array}$

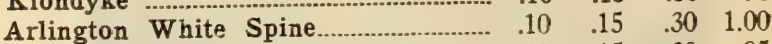

$\begin{array}{llllll}\text { Improved Extra Early White Spine } & .10 & .15 & .30 & .95\end{array}$ 


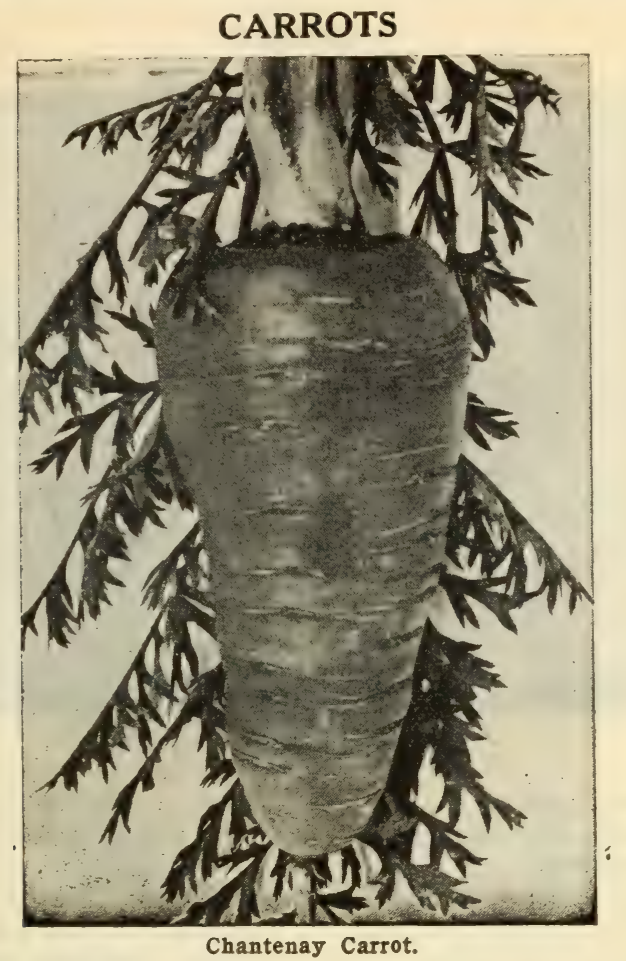

CHANTENAY. For a general crop the best; the largest yield; the most profitable for the market gardeners. Flesh sweet, crisp and tender. It is a desirable second early Carrot for the home garden and is also suitable for field culture. Pkt. $10 \mathrm{c}$; 0z. $15 \mathrm{c} ; \mathrm{r} / 4 \mathrm{lb} .30 \mathrm{c}$; 1b. $85 \mathrm{c}$. DANVERS. Medium early. Pkt. 10c; oz. 15c; $1 / 4$ lb. $30 \mathrm{c}$; 1b. $85 \mathrm{c}$.

OXHEART. Early. Pkt. 10c; 0z. 15c; 1/4 lb. 30c; lb. $90 \mathrm{c}$.

EARLY RUBICON. Pkt. 10c; 0z. 15c; $1 / 4$ lb. 35c; Ib. $\$ 1.00$.

\section{KALE}

GREEN CURLED SCOTCH, or NORFOLK. Pkt. 10c; oz. $15 \mathrm{c} ; 1 / 4 \mathrm{lb} .25 \mathrm{c} ; 1 \mathrm{lb} .85 \mathrm{c}$.

IMPERIAL. Pkt. $10 \mathrm{c}$; 0z. $15 \mathrm{c}$; $1 / 4$ lb. $25 \mathrm{c} ; 1 \mathrm{lb} .75 \mathrm{c}$. SIBERIAN. Pkt. $10 \mathrm{c}$; oz. $15 \mathrm{c} ; \mathrm{l} / 4 \mathrm{lb} .20 \mathrm{c} ; \mathrm{lb} .50 \mathrm{c}$.

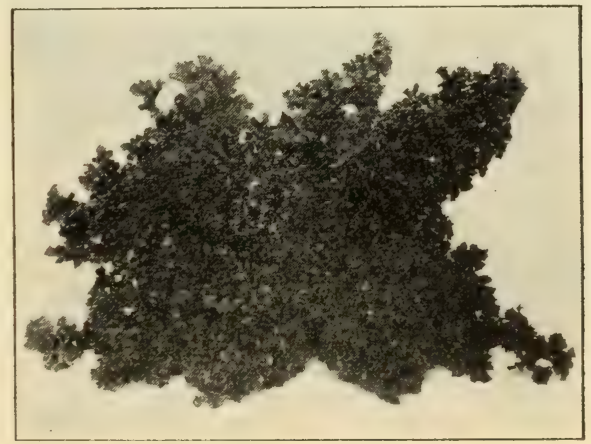




\section{CABBAGE}

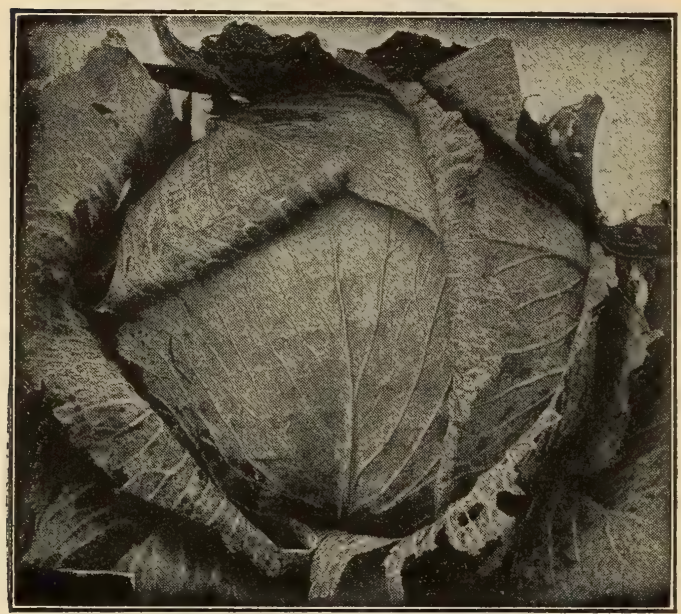

Bolgiano's New Early Cabbage.

NEW EARLY. A favorite with the market gardeners. Pkt. 10 ; oz. $25 \mathrm{c} ; \mathrm{I} / 4$ lb. $75 \mathrm{c} ; 1$ lb. $\$ 3.00 ; 5$ lbs. $\$ 11.00$. All postpaid.

CHARLESTON LARGE WAKEFIELD. Resembles the Early Jersey Wakefieid in form but it is much larger. Very desirable for the market gardener. Pkt. 10c; oz. 25c; I $/ 4$ lb. $75 \mathrm{c} ; 1$ lb. $\$ 2.75 ; 5$ lbs. $\$ 10.50$. All postpaid.

SUCCESSION. A very good variety for second early use. Makes good sized flat heads and is very tender and of fine grain. Pkt. 10c; oz. 25c; $1 / 4 \mathrm{lb} .75 \mathrm{c} ; 1 \mathrm{lb}$. $\$ 2.50 ; 5$ lbs. $\$ 10.50$. All postpaid.

Pkt. Oz. $1 / 4 \mathrm{lb} . \mathrm{Lb}$.

Big Winner Wakefield $\$ 0.10 \$ 0.25 \$ 0.80 \$ 3.00$

Early Jersey Wakefield $\begin{array}{llll}.10 & .25 & .75 & 2.50\end{array}$ Copenhagen Market

$\begin{array}{llll}.10 & .25 & .85 & 3.00\end{array}$

Early Flat Dutch $\quad 10 \quad 25$

Square Deal ……….............................. $\quad .10 \quad .25$

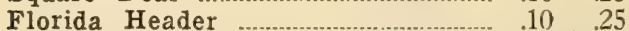

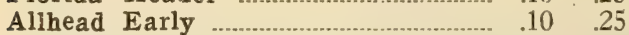

All Season ..................................................

Extra Large Flat Dutch..................... $\quad .10 \quad .25$

Danish Ball Head Short Stem ............. $.10 \quad .25$

Drumhead Savoy ................................ $\quad .10 \quad .25$

Marnmoth Red Rock $\ldots$

\section{COLLARDS}

True Georgia Southern.

F. W. Bolgiano \& Co.

I sent you an order a few years ago for your Purple Top White Globe Turnips, and I never had any better turnips than they were. Small tops and fine shaped bulbs. Please send me the same kind you sent me then.

Yours very truly,

$$
\text { GEORGE T. ROBINSON, }
$$




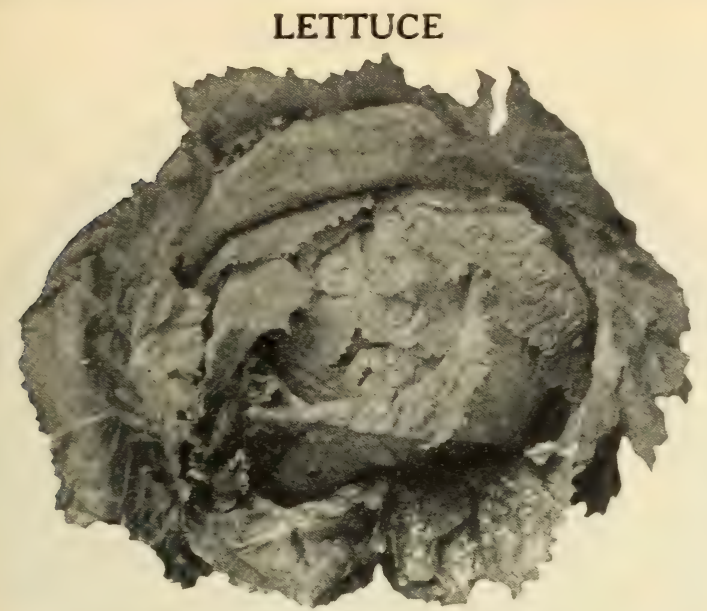

BIG BOSTON. Bolgiano's Famous Stock. Pkt. 10c; 0z. $15 \mathrm{c} ; \mathrm{I} / 4 \mathrm{lb} .45 \mathrm{c} ; 1 \mathrm{~b} . \$ 1.50$.

LOS ANGELES. Large, late. Pkt. 10c; oz. 25c; I $/ 4$ lb. $80 \mathrm{c} ;$ lb. $\$ 2.75$. Postpaid.

T R I A N O N COS. Pkt. 10c; oz. 15c; I/4 lb. 45c; 1 lb. $\$ 1.50$.

Pkt. Oz. I $/ 4 \mathrm{lb}$. Lb.

MASTERPIECE ………………...... $\$ 0.10 \$ 0.35 \$ 1.15 \$ 4.00$

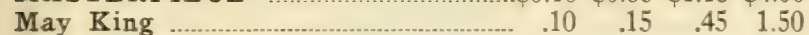

$\begin{array}{lllll}\text { New York or Wonderful } \ldots \ldots \ldots \ldots \ldots \ldots \ldots \ldots & .10 & .25 & .80 & 2.75\end{array}$

$\begin{array}{lllll}\text { Hanson } & 10 & .15 & .45 & 1.50\end{array}$

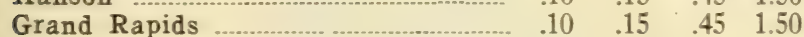

$\begin{array}{lllll}\text { Iceberg _ } ~ 10 & .15 & .45 & 1.50\end{array}$

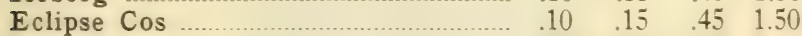

Salamander $\ldots \ldots \ldots \ldots \ldots \ldots \ldots \ldots \ldots \ldots \ldots n$

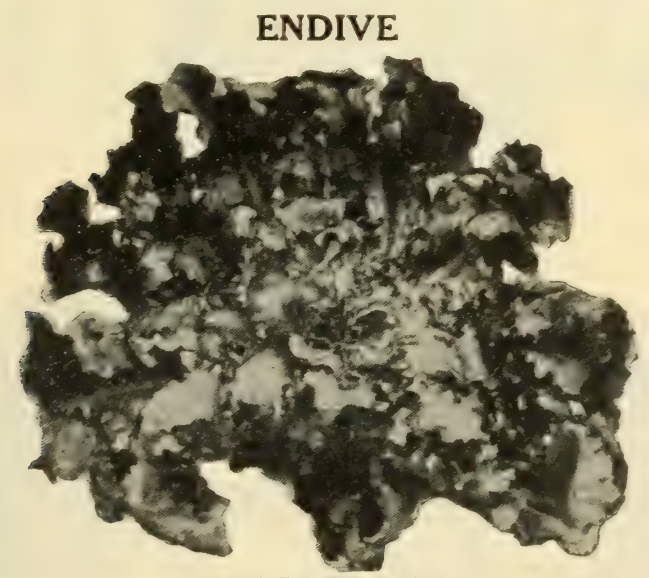

Broad Leaf Batavian.

Pkt. Oz. $1 / 4 \mathrm{lb} . \mathrm{Lb}$.

White Curled Self-Blanching …........ $\$ 0.10 \$ 0.15 \$ 0.50 \$ 1.75$

Large Green Curled ……........................ $\quad .10 \quad .15 \quad .45 \quad 1.50$

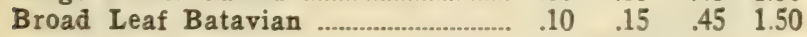

MUSTARD

$\begin{array}{lllll}\text { Southern Giant Curled …........................ } & .10 & .15 & .25 & .75\end{array}$

$\begin{array}{lllll}\text { Chinese Broad Leaf } \ldots \ldots \ldots \ldots \ldots \ldots \ldots \ldots \ldots & .10 & .15 & .35 & 10\end{array}$ 


\section{MUSKMELON}

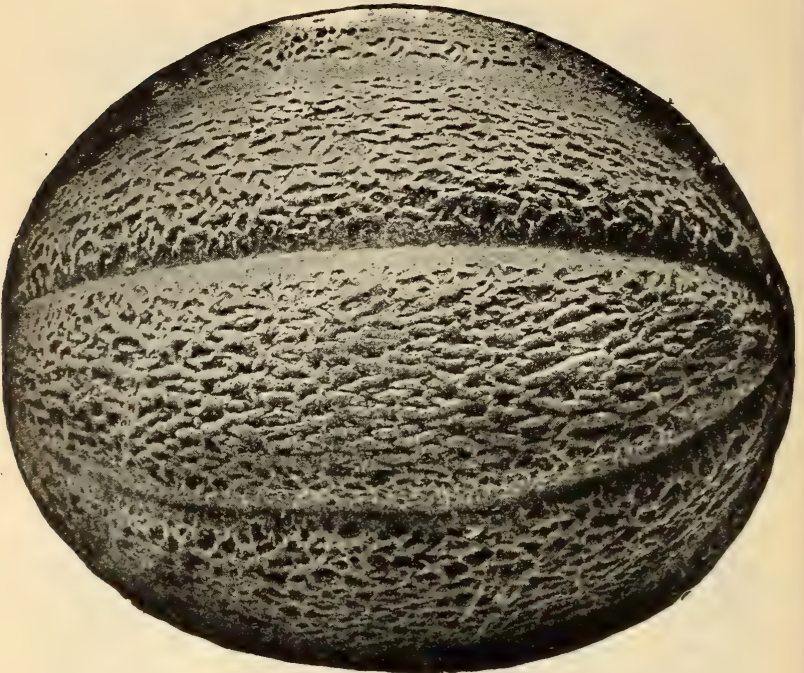

Pkt. Oz. $1 / 4$ Lb. Lb.

Sweet Air $\$ 0.10 \$ 0.15 \$ 0.45 \$ 1.50$

Rockyford

$\begin{array}{llll}.10 & .15 & .30 & .90\end{array}$

Junior Rockyford

$\begin{array}{llll}.10 & .15 & .50 & 1.75\end{array}$

Unsurpassed

$\begin{array}{lll}.20 & .60 & 2.00\end{array}$

Knight

$.10 \quad .20$

Baltimore Nutmeg

$\begin{array}{llll}.10 & .15 & .35 & 1.25 \\ & .15 & .35 & 1.00\end{array}$

WATERMELON

General Pershing

Tom Watson

Irish Gray

Florida Favorite

Kleckley Sweet

Excell

$\begin{array}{rrrr}.10 & .15 & .35 & 1.00 \\ .10 & .15 & .25 & .75 \\ .10 & .15 & .25 & .85 \\ .10 & .15 & .25 & .75 \\ .10 & .15 & .25 & .75 \\ .10 & .15 & .25 & .85\end{array}$

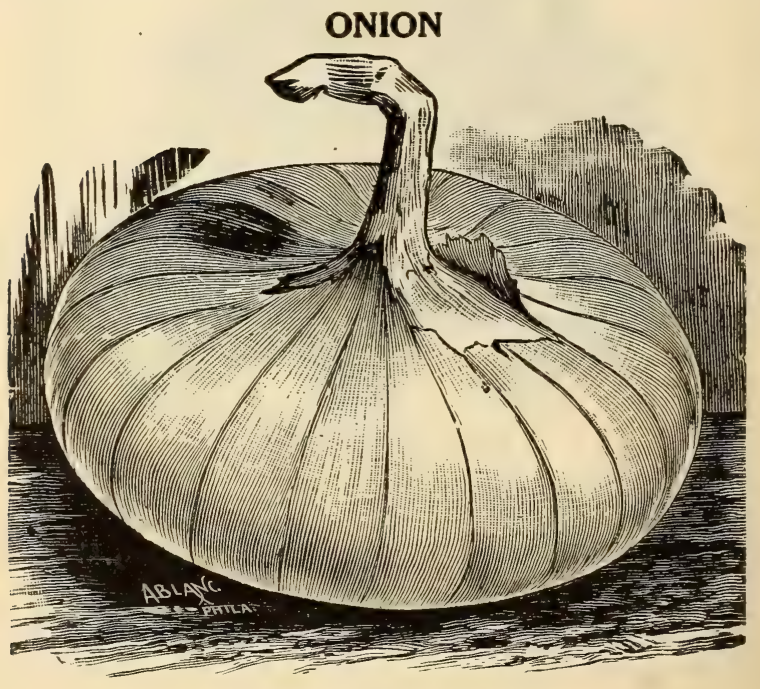

White Silver Skin Onion.

(18) 


\section{ONIONS-Continued}

Pkt. Oz. I/4 Lb. Lb.

White Silver Skin

$\$ 0.10 \$ 0.30 \$ 1.00 \$ 3.50$

Southport White Globe $\ldots . .10 \quad .35 \quad 1.15 \quad 4.00$

$\begin{array}{lllll}\text { Southport Yellow Globe } \ldots \ldots \ldots \ldots-\ldots . .3 & .10 & .35 & 1.15 & 4.00\end{array}$

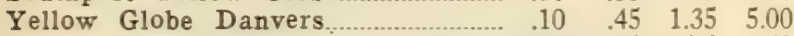

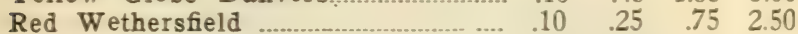

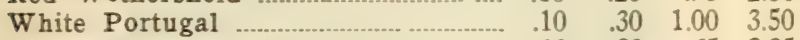

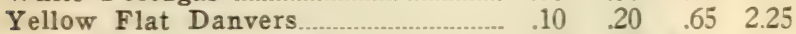

Crystal Wax Bermuda ……..................... $\quad .10 \quad .45 \quad 1.50 \quad 5.50$

White Bermuda ................................... Please write for prices

Red Bermuda .................................... Please write for prices

\section{PEAS}

LAXTONIAN. Extremely early; wrinkled Pea. Peas very sweet; deep green. Pkt. $10 \mathrm{c}$; pt. $25 \mathrm{c}$; g.t. $45 \mathrm{c}$; pk. $\$ 3.15$; bu. $\$ 11.50$.

THOMAS LAXTON. Pkt. 10c; pt. 25c; qt. 45c; pk. $\$ 2.75$; bu. $\$ 10.00$.

GRADUS. Pkt. $10 \mathrm{c} ; \quad$ pt. $25 \mathrm{c} ; \quad$ qt. $45 \mathrm{c} ;$ pk. $\$ 2.75$; bu. $\$ 10.00$.

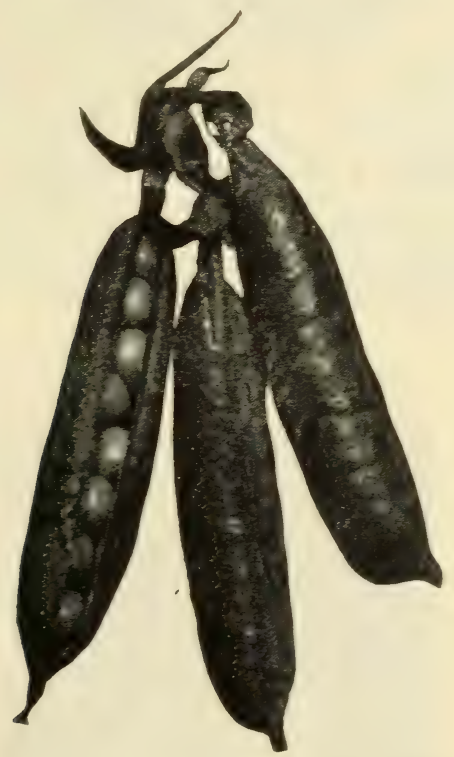

Pt. Qt. Pk. Bu.

Early Bird

$\$ 0.30 \$ 0.50 \$ 3.50 \$ 12.00$

Early Springtime

$\begin{array}{llll}.25 & .45 & 3.15 & 11.50\end{array}$

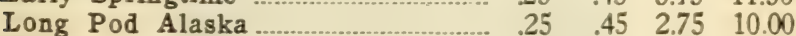

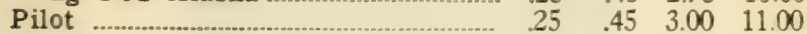

$\begin{array}{llllll}\text { Extra Early Alaska } \ldots \ldots \ldots \ldots \ldots \ldots & .25 & .40 & 2.50 & 9.00\end{array}$

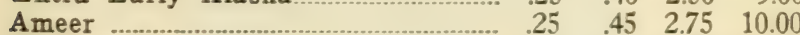

$\begin{array}{lllll}\text { First and Best } \ldots \ldots \ldots \ldots \ldots \ldots \ldots \ldots \ldots \ldots n & .25 & .45 & 2.50 & 9.00\end{array}$

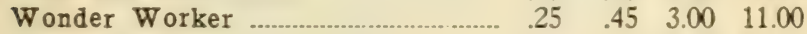

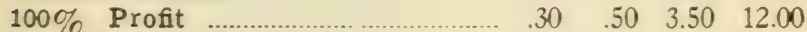

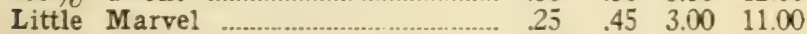

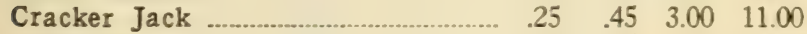

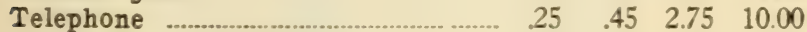

Champion of England $\ldots \begin{array}{lllll}.25 & .45 & 2.50 & 9.00\end{array}$

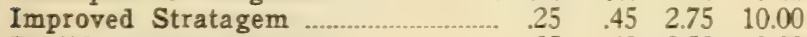

$\begin{array}{lllll}\text { Prolific Early Market.....____ } & .25 & .40 & 2.50 & 9.00\end{array}$

(19) 


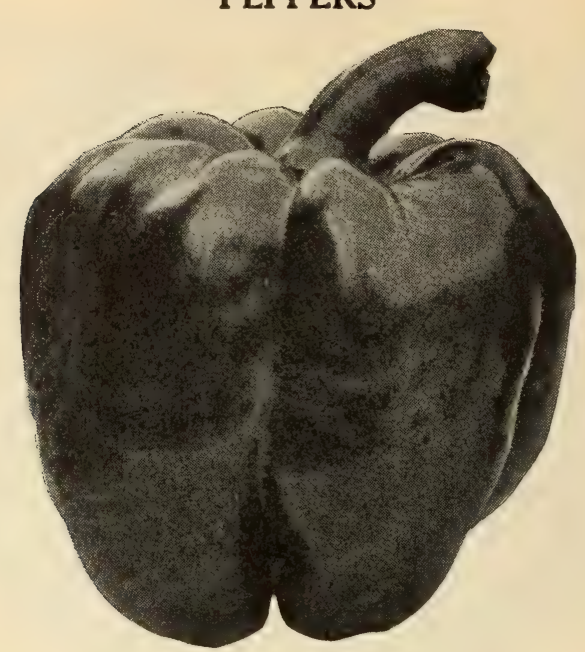

ROYAL KING. Pkt. 10c; 0z. 30c; 1/4 lb. \$1.00; 1b. \$3.50. CHINESE GIANT. Pkt. 10c; oz. 45c; $1 / 4$ lb. $\$ 1.35$; 1b. $\$ 5.00$.

RUBY KING. Large, scarlet fruit; rather mild flavor. Pkt. 10c; 0z. 25c; 1/4 lb. 75c; 1b. $\$ 2.50$.

WORLD BEATER. Pkt. 10c; oz. 30c; $1 / 4$ lb. $\$ 1.00$; 1b. $\$ 3.50$.

PERFECTION. Pimento. Pkt. $10 \mathrm{c} ;$ oz. $25 \mathrm{c} ; \mathrm{I} / 4 \mathrm{lb}$. $85 \mathrm{c}$; 1b. $\$ 3.00$. Postpaid.

\section{PARSLEY}

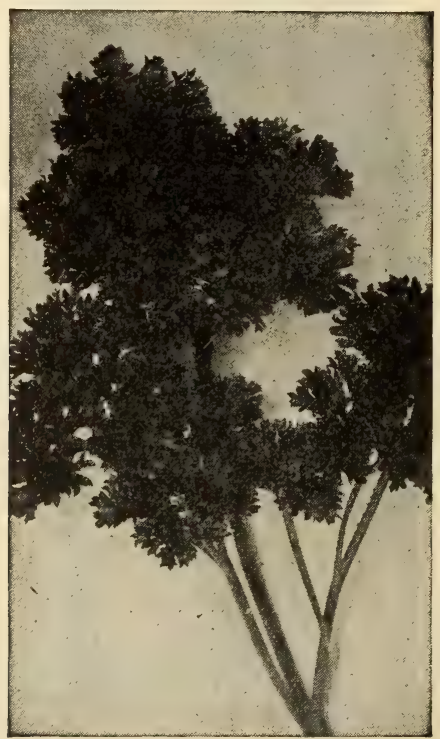

MARKET GARDENER'S TRIPLE CURLED. EXceedingly large yielder. Stands heat and cold. Pkt. 10c; oz. $15 \mathrm{c} ; \mathrm{I} / 4$ lb. $30 \mathrm{c}$; lb. $75 \mathrm{c}$. All postpaid. 


\section{RADISHES}

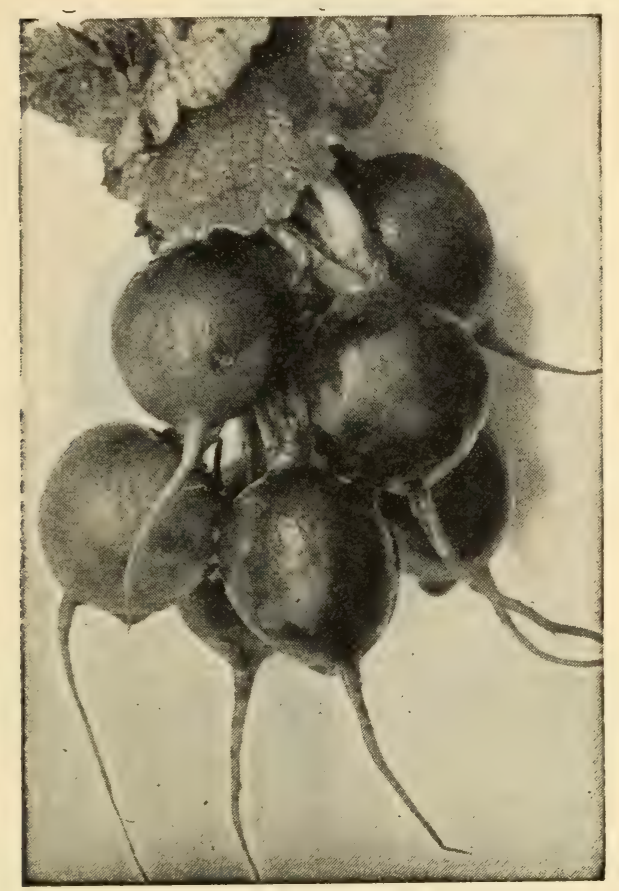

EARLY SCARLET GLOBE. A splendid extra early. round, bright red radish. Pkt. 10c; oz. 15c; $1 / 4$ lb. 25cy 1b. 75c. All postpaid.

WHITE TIP SCARLET TURNIP. Pkt. 10c; oz. 15c; $1 / 4$ lb. $25 \mathrm{c}$; 1b. $85 \mathrm{c}$.

EARLY LONG SCARLET SHORT TOP. Pkt. 10c; oz. $15 \mathrm{c} ; 1 / 4$ lb. $25 \mathrm{c}$; 1b. $75 \mathrm{c}$.

LONG RED. The roots are slender and are often six to seven inches long. Pkt. 10c; $1 / 4$ lb. 25c; lb. $75 \mathrm{c}$.

ICICLE. Long White. Pkt. 10c; 1/4 lb. 25c; 1b. $75 \mathrm{c}$;

5 ibs. \$3.25. All postpaid.

WHITE CHINESE WINTER. Pkt. 10c; 0z. 15c; $1 / 4$ lb. $25 \mathrm{c}$; lb. $75 \mathrm{c}$.

CHINESE ROSE WINTER. Pkt. 10c; 0z. 15c; I/4 lb. $25 \mathrm{c} ;$ lb. $75 \mathrm{c}$.

\section{SPINACH}

BOLGIANO'S SELECTED BLOOMSDALE SAVOY. A mammoth seeded selection that possesses a vitality and vigor of growth and far outyields any other variety. After many years of tireless effort at breeding we are pleased to be able to offer a limited quantity of this improvement on the old standby. Productive, tender and excellent quality. Oz. $10 \mathrm{c} ; \mathrm{I} / 4$ lb. $15 \mathrm{c} ; 1 \mathrm{~b} .35 \mathrm{c} ; 5 \mathrm{lbs}$. \$1.25. All postpaid.

NEW SPRING. Oz. 10c; $1 / 4$ 1b. 15c; 1b. 30c; 5 lbs. \$1.25. All postpaid.

VICTORIA. Oz. 10c; I/4 lb. 15c; 1b. 30c; 5 lbs. $\$ 1.25$. All postpaid. 


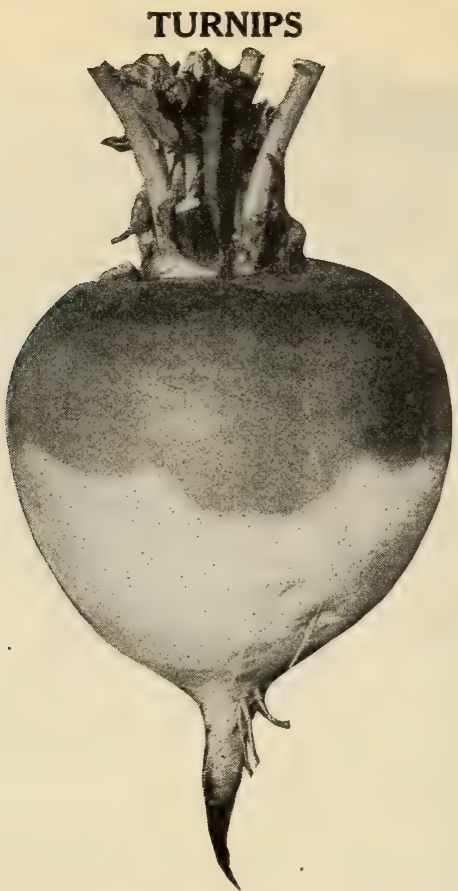

PURPLE TOP WHITE GLOBE. Superior quality. A heavy cropper, early, and a fine keeper. Pkt. 10c; 0z. 15c; 1/4 lb. 20c; 1b. 50c; 5 lbs. \$2.75. All Postpaid.

EARLY SNOWBALL. Very fine. Pkt. 10c; 0z. 15c; $1 / 4$ lb. 20c; 1b. 50c.

GOLDEN BALL. Yellow, early. Pkt. 10c; oz. 15c; I/4 lb. 20c; 1b. 50c. Pkt. Oz. I/4 Lb. Lb. Extra Early Purple Top Milan.......\$0.10 \$0.15 \$0.20 \$0.95 $\begin{array}{lllll}\text { Purple Top White Flat Strap Leaf.... } & .10 & .15 & .20 & .50\end{array}$ $\begin{array}{lllll}\text { White Flat Dutch Extra Early........... } & .10 & .15 & .20 & .40\end{array}$ Green Top Yellow Aberdeen ..................

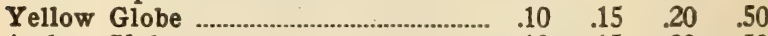

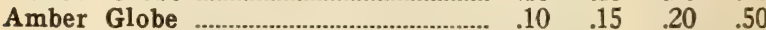

RUTABAGA-LONG ISLAND IMPROVED PURPLE

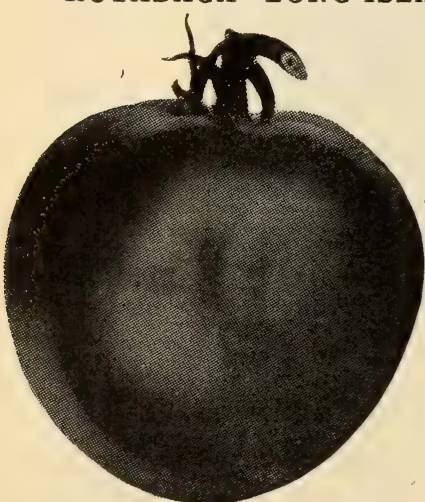

TOP. Pkt. 10c; oz. 15c;

$1 / 4$ lb. $25 \mathrm{c}$; 1b. $75 \mathrm{c}$.

\section{TOMATOES}

WASHINGTON, Pkt. $25 \mathrm{c} ;$ oz. $\$ 2.25 ; \mathrm{x} / 4$ lb. $\$ 7.50$; lb. $\$ 25.00$.

IMPROVED STONE. Pkt. $10 \mathrm{c}$; 0z. $25 \mathrm{c} ; \mathrm{I} / 4$ lb. $85 \mathrm{c}$; lb. $\$ 3.00$.

GREATER BALTIMORE. Pkt. 10c; oz. 35c; $\mathrm{I} / 4$ lb. $\$ 1.15 ; \mathrm{lb} . \$ 4.00$.

FLORIDA SPECIAL. Blight resistant. Pkt. 10c; oz. $40 \mathrm{c} ; \quad \mathrm{T} / 4 \quad \mathrm{lb}$. $\$ 1.40$; lb. $\$ 4.50$. Postpaid.

LIVINGSTON'S GLOBE. Purple-red. Pkt. 10c; oz. $35 \mathrm{c} ; \mathrm{I} / 4$ lb. $\$ 1.25 ; \mathrm{lb} . \$ 4.00$. 


\section{BOLGIANO'S “CAPITOL PARK” LAWN GRASS SEED}

Has Greatly Helped to Make Washington the City Beautiful Used in Seeding Many of the Handsome Lawns Surrounding the Government Buildings. It Never Fails to Give Satisfaction.

BOLGIANO'S "CAPITOL PARK” LAWN GRASS SEED, from yearly.comparative trials, has proven to be unsurpassed for first-class and permanent results. This is due to the fact that we use only the very finest varieties of the most suitable grasses, thoroughly recleaned, free from all chaff and weed seeds.

BOLGIANO'S "CAPITOL PARK" LAWN GRASS SEED contains a well-balanced blend of various American and foreign fine bladed and deeply rooting grasses which has proved to produce the very best results under the varied conditions as to soil and climate met with in America.

BOLGIANO'S "CAPITOL PARK" LAWN GRASS SEED will give a green, smooth, velvety sod with a thick bottom and free from such varieties of common grasses as produce clumps and knots on the lawn, which so frequently spoil the desired smooth and deep effect.

Pkt. $10 \mathrm{c} ; 1$ lb. $40 \mathrm{c} ; 10$ lbs. $\$ 3.50 ; 100$ lbs. $\$ 32.50$.

\section{BOLGIANO'S “SHADY NOOK” LAWN GRASS SEED}

\section{The Grass That Will Grow in Shady Places}

More than twenty years of unremitting toil has been rewarded with success in the SHADY NOOK LAWN GRASS SEED. Not until the spring of 1905 did we succeed in accomplishing that for which we set out. Time and again we thought we had, but only to realize that failure was our portion. The SHADY SIDE of the STREET and under the OLD OAK TREE; in fact, everywhere that the sun does not strike more than a few minutes each day-those are the places that we want to make pretty for you. Pkt. 10c; lb. 40c; 10 lbs. $\$ 3.50$; 100 lbs. $\$ 32.50$.

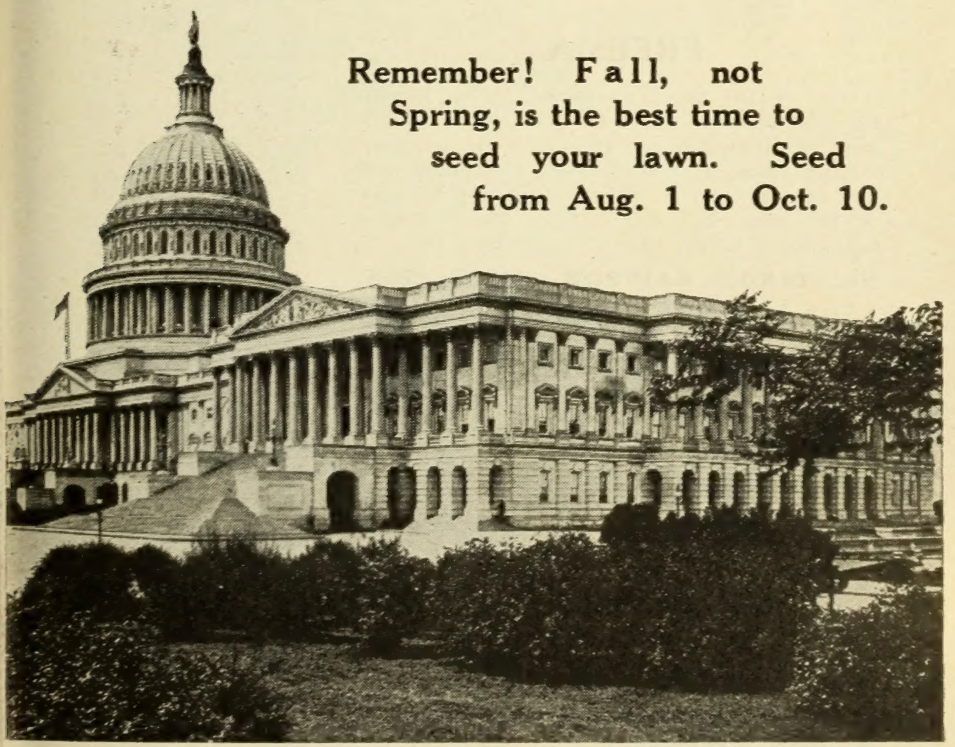

(23) 



\section{FIELD SEED AND POTATO PRICE}

6293

JANUARY 20, 1926.

Al prices quoted here give the values prevailing on th list was published. We do not anticipate any great changes thi but should the prices be more or less when your order is recir will de sent the full value of your money at prices then preva A new copy of this list with the latest prices will be sent or at any time.

\section{CORN}

Prosperity

Reid's yeliow Dent

Leaming:

Choice Yellow Dent

Boone Jo. White

Hickory King

Eureka Enilage

Jhoice White Dent

MISCELLANEOUS

White Spring Oats

Hairy Vetch
Spring
D. E. Rape
Tennessee Millett
Japan Buckwheat
Uanada Peas
Evergreen Broom Corn

\section{SOY BEANS}

Nammoth Yellow

Nammoth BIa k

Wilson Early

$\mathrm{Va}$.

Trust Buster

Irish Cobblers

Early jose

Early Chio

Red BIiss Triumur

Prosperity

Areei. Mountain

palding

American Giants

Bur ank Seedling

Eirıy Kound Six Weess

E.jign Bagley

$\mathrm{P} \perp$ u. Ky Baltimore

iray's Mortgage lifter

McCormiak
10 LBS. 50 LBS. CLOVERS

10 LBS

$\begin{array}{cc}.75 & 3.00 \\ .65 & 2.50 \\ .05 & 2.50 \\ .00 & 2.25 \\ .65 & 2.50 \\ .65 & 2.75 \\ .65 & 2.50 \\ .60 & 2.25\end{array}$

.50

1.00

1.60

1.15

1.50

1.00

.50

.75

1.50

$\begin{array}{ll}.6 C & 225 \\ 60 & 2.25 \\ .65 & 2.75 \\ .05 & 2.75\end{array}$

ALFALFA
Sapling
Medium Red
Crimson
Alsike
White
White Sweet
Japar

2.50

4.00

4.00

1.10

3.10

5.00

1.50

2.50

\section{GRASSES}

Kentucky Blue

4.50

TIMOTHY

Red Top

Orchard

Sudan

Perennial kyt

Ita.lian"

Creeping Bent

Sheep Fescue

Red Fescue

Sorghum

COW PEAS

Whippoorwill

Clay

"ixed

\section{POTATOES}

$\mathrm{Bbl}$.

10.75

9.50

10.00

10.00

10.00

1050

9.50

10.00

10.00

9.75

9.75

9.75

9.75

9.75

9.00

\section{Bu.}

5.25

4.85

5.00

5.00

5.00

5.25

4.85

5.00

5.00

4.85

4.85

4.85

4.85

4.85

4.50
Peck.

1.50

1. 25

1.25

1.25

1.25

1.50

1.25

1.25

1. 25

1.25

1. 25

1. 25

1.25

1.25 


\section{F. W. BOLGIANO \& CO.}

Growers of Seeds that Succeed

1009 B Street, N. W., Washington, D. C.

Evergbody Can Raise Them. Grown Among Pebbles in Bowls.

\section{PAPER WHITE NARCISSUS}

Ready for Delivery Late in September

These beautiful pure white flowers with their delicious odor are the most charming and easily grown of indoor plants. They will provide a table decoration from Christmas to Easter planted at different times. As a Christmas gift a dozen bulbs in a bowl make a gift both unique and beautiful in sentiment.

PRICE : Each ...................... \$0.10

Per dozen ................. 1.00

Per hundred ............... 7.50

\section{FREESIA}

One of the most charming bulbs known for Winter-flowering and cut flowers. They grow well and bloom freely in an ordinary sitting room, if placed near a window and not kept too warm. They need not be kept in the dark as other bulbs. Plant 4 to 6 bulbs in a 5-inch pot, in light, rich soil.

\section{BOLGIANO'S RAINBOW COLLECTION.} Flowers two inches long, borne in clusters of 6 to 10 . The body of the flower is pure white with lower segments spotted lemonyellow. The perfume is most delicious. Dozen 70c; $100, \$ 5.00$.

\section{PlanTabbs}

\section{ODORLESS PLANT FOOD}

PlanTabbs wonderfully assists in the growing of bulbs for either indoor or outdoor culture. Easily used. Just stick one tablet in the ground each week a short distance from the bulb and watch the wonderful results. 30 Tablets, $25 \mathrm{c}$; 65 Tablets, 50c; 150 Tablets, $\$ 1.00$.

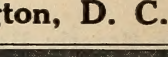

